FREE FIELD THEORIES OF SPIN-MASS TRA ETCTORIES AND QUANTUM ELECTRODYNAMICS IN THE NULL PLANE

By

George R. Bart and Stanley Fenster

June 1976

MASTER

DISTRIBUTION RE IHESS ROCUIMENT IS LNLMUTED

ARGONNE NATIONAL LABORATORY, ARGONNE, ILLINOIS

operated under contract W-31-109-Eng-38 for the U. S. ENERGY RESEARCH AND DEVELOPMENT ADMINISTRATION 
The facilities of Argonne National Laboratory are owned by the United States Government. Under the terms of a contract (W-31-109-Eng-38) between the U. S. Energy Research and Deveiopment Administration, Argonne Universities Association and The University of Chicago. the University employs the staff and operates the Laboratory in accordance with policies and programs formulated, approved and reviewed by the Association.

\section{MEMBERS OF ARGONNE UNIVERSITIES ASSOCIATION}

The University of Arizona Carnegie-Mellon University Case Western Reserve University The University of Chicago University of Cincinnati Illinois Institute of Technology University of Illinois Indiana University Iowa State University The University of lowa
Kansas State University The University of Kansas Loyola University Marquette University Michigan State University The University of Michigan University of Minnesota University of Missouri Northwestern University University of Notre Dame
The Ohio State University Ohio University

The Pennsylvania State University

Purdue University

Saint Louis University Southern Illinois University The University of Texas at Austin Washington University Wayne State University The University of Wisconsin

\section{NOTICE}

This report was prepared as an account of work sponsored by the United States Government. Neither the United States nor the United States Energy Research and Development Administiation, nor any of their employees, nor any of their contractors, subcontractors, or their employees, makes any warranty, express or implied, or assumes any legal liability or responsibility for the accuracy, completeness or usefulness of any information, apparatus, product or process disclosed, or represents that its use would not infringe privately-owned rights. Mention of commercial products, their manufacturers, or their suppliers in this publication does not imply or connote approval or disapproval of the product by Argonne National Laboratory or the U. S. Energy Research and Development Administration. 


\title{
FREE FIELD THEORIES OF SPIN-MUSS TRAJECTORIES AND QUANTUM ELECTRODYNAMICS IN THE MULL PLANE
}

\author{
George R. Bart \\ Northeast College \\ Chicago, Illinois 60640 \\ and \\ Stanley Fenster * \\ High Energy Physics Divioion \\ Argonne National Laboratory \\ Argonne, Illino1s 60439
}

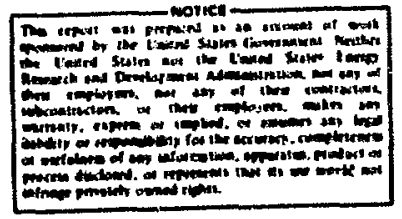

FWork performed under the auspices of the United States Bnergy Research and Development Administration. 


\section{ABSTRACT}

The ten generators of the Poincaré algebra for quantum electrodynamics and other gauge theories are given in the null plane. The explicit correspondence of thefr field-theoretic form to the Bacry-Chang group-theoretic form in the free case is pointed out. It is then noticed that the forms are independent of the spin and allow inclusion of charge quantum numbers at will, which indicates that they represent an advantageous free-particle starting point for a hadron theory with positive spin-mass trajectories (SMr) and with interaction. The internal oscillator content is extracted for both gauge theories and dual resonance models. Interactions are cublc and quartic in the fields. In the dual model they encompass the SIT, wheress no straightforward extension to Sirt is possible for the manifesty covartant theories. The requirements of a fieldtheoretic SWr interaction are spelled out in an algebraic form which guarantees Poincare invariance; however no such interaction is yet known. The approach indicates how a realistic spectrum might, be achieved without composite hadrons and incorporating full Poincare invariance. 
I. Introduction

Experiment indicates that hadron resoriances may come in Infinite spin-mass trajectories. Unhappily, the construction of a rigorous realistic theory of interacting trajectories seems to be an arduous task. In the years 1966-68 infinite component wave equarions were examined and attempts were made to fit their apectra to hadrons ${ }^{l}$ and to the hydrogen atom. ${ }^{2}$ The latter succeeded but the former suffered from space-like solutions ${ }^{3}$ and/or unrealiatic spin-mass spectro. It was never proved, however, that these disadvantages could not be overcome by more complex second order equations. ${ }^{4}$ Contemporaneously, the infinite momentum frame method was discovered ${ }^{5}$ and its relation to Dirac's "front form" realized. 6 Some theorlsts comprehended that the null plane (NP) approach might lead to a relativistically covariant theory containing a positive mass spectrum. ${ }^{7}$ Thus, NP field theory was devised and applied to $Q E D^{8-11}$ to a $\bar{\psi} \psi \phi$ model ${ }^{12}$, to fermionmassive boson theories, 13,14 and to Yang-Nills theories 15,16 . Advantages of the NP approach were clearly perceived in this work. For example, in numerical QED calculations of the fourthorder contributions to the magnetic moment of the electron, the inherent elimination of redundant field components and the avoidance of subsidiary conditions reduced the cormuter time by a factor 2 to 5.17

Although the experimental evidence for purely linear Regge trajectories is meager, they are an appealing simplification from some theoretical viewpoints. In fact, in the dual model, 
which evolved during the last decade, they are an essential ingredient. 18 Several authors have analysed the dual model as a NP field theory ${ }^{19-21}$ - the first one to incorporate infinitely rising trajectories. Relativistic covariance was proved ingeniously. 22 The method of proof, however, did not exhibit ten quantized Poincare group generators with interaction terms and without redundant components. No one could incorporate currents with rigor and it seemed that 26 transverse space dimensions were required. 23,24

Also in this period, Dirac and others advanced first quantized models having linearly rising positive spin-mass spectra. 25 A NP formulation of the Dirac model hiss devised ar:interpreted as describing a composite relativistic object possessing two constituents interacting via action-at-a-distance forces.

In this paper we illustrate how a NP prescription elegantly describes trajectories with positive mass spectra. First, however, we examine some aspects of NP technique which seem likely to provide a theory of hadrons. In Section II, the universal form of the Poincare generators appropriate to the NP is deriver. 9,26,27 In Section III, all standard free field theories are reduced to a second quantized version of the universal form. It is pointed out that the number of field components does not appear. Since fields for infinitely rising trajectories must have an Infinite number of components, this is a crucial advantage in the construction of a trajectory field theory. 
In Section IV, a free field theory of spin-mass trajectories is constructed by invoking the method of dynamical groups to replace the mass-squared and the spin symbols by products of harmonic oscillators. Several examples of internal spaces are mentioned, ranging from the simplest, the Dirac model, to the most complex, the dual model. In Section $V$, we show how the isospin type groups can be introduced easily; we describe how the SU(6) $\times 0$ (3) model of hadrons is naturally built with the NP Hamiltonian technique. In Section VI, the modification of the Poincaré generators is discussed when interaction is present. Vertices and seagulls are presented for QED and the general. kinematic structure is described. The zero slope limit of the dual model vertex is calculated and compared with those of standard field theories. The dual model seagull term has not yet been explicitly deduced but should be extractable from a certain known diagram. Section VII is a statement of the algebraic problem posed by a hadron theory in NP form. In Appendix A we derive the form of the internal coordinate angular momentum operators $j$ in terms the components of the PauliLubanski vector $W_{\mu}$. The reduction of a vector gluon field theory to NP form is sketched in Appendix B. 
II. Null Plane Representation of the Poincare Generators

Denote the generators in covariant tensorial form by $P_{\mu}, M_{\mu \nu}(\mu-0,1,2,3)$ with metric $\left(g_{00}, g_{11}, g_{22}, g_{33}\right)=$

$(1,-1,-1,-1)$. The commutation relations are:

$$
\begin{aligned}
& {\left[P_{\mu}, P_{\nu}\right]=0, \quad\left[M_{\mu \nu}, P_{\rho}\right]=1 g_{\nu \rho} P_{\mu}-1 g_{\mu \rho} P_{\nu}} \\
& {\left[M_{\mu \nu}, M_{\rho K}\right]=i g_{\nu \rho} M_{\mu K}+1 g_{\mu K} \dot{M}_{\nu \rho}-i g_{\nu K} M_{\mu \rho}-1 g_{\mu \rho} M_{\nu K} .}
\end{aligned}
$$

The Pauli-Lubanski vector is the sum of products

$$
W_{\mu}=-\frac{1}{2} \varepsilon_{\mu \nu K \lambda} P \nu_{M}{ }^{k \lambda}
$$

obeying

$$
\begin{aligned}
& {\left[W_{\mu}, P_{\nu}\right]=0, \quad\left[M_{\mu \nu}, W_{\lambda}\right]=1 g_{\nu \lambda} W_{\mu}-1 g_{\mu \lambda} W_{\nu}} \\
& {\left[W_{\mu}, W_{\nu}\right]=1 \varepsilon_{\mu \nu \rho \sigma} W^{P_{P} \sigma} .}
\end{aligned}
$$

The Casimir operators are $P^{2}$ and $W^{2}$ and it is true that $W \cdot P=0$. It is convenient to introduce three-vectors $J_{a}, K_{a}$ via

$$
M_{a b}=\varepsilon_{a b c} J_{c}, M_{a o}=K_{a} \quad(a=1,2,3)
$$

satisfying

$$
\begin{aligned}
& {\left[J_{a}, J_{b}\right]=i \varepsilon_{a b c} J_{c},\left[J_{a}, K_{b}\right]=i \varepsilon_{a b c} K_{c}} \\
& {\left[K_{a}, K_{b}\right]=-i \varepsilon_{a b c} J_{c}}
\end{aligned}
$$

in terms of which

$$
W_{0}=P_{a} J_{a}, \quad W_{a}=P_{o} J_{a}+\varepsilon_{a b c} P_{b} K_{c} .
$$

These vectors are stepping stones to the special combinations used in null plane.

Unfortunately there are a variety of notations and normalizations used in the literature for these quantities. Our choice emerges from a unitary transformation selected so that the metric $g_{\mu \nu}$ is transformed to 


$$
g_{\mu \nu}=U_{\mu}{ }^{P} g_{\rho \sigma} v_{\nu}^{v}=g^{\mu \nu}=\left(\begin{array}{rrrr}
0 & 0 & 0 & 1 \\
0 & -1 & 0 & 0 \\
0 & 0 & -1 & 0 \\
1 & 0 & 0 & 0
\end{array}\right)
$$

where the real matrix $U$ is given by

$$
U_{\nu}^{\mu}=\left(\begin{array}{rrrr}
1 / \sqrt{2} & 0 & 0 & 1 / \sqrt{2} \\
0 & 1 & 0 & 0 \\
0 & 0 & 1 & 0 \\
1 / \sqrt{2} & 0 & 0 & -1 / \sqrt{2}
\end{array}\right) .
$$

Under this transformation a vector $A^{\mu}$ is transformed to $\hat{A}^{\mu}=U^{\mu}{ }_{V} A^{\nu}$. To avoid an excess of carets, it is convenient to use $A^{+}, A^{-}, A_{-}, A_{+}$instead of $\hat{A}^{0}, \hat{A}^{3}, \hat{A}_{0}, \hat{A}_{3}$ respectively. Thus $A^{+}=A_{-}=\hat{A}^{0}=\hat{A}_{3}=\left(A^{0}+A^{3}\right) / \sqrt{2}$ and $A^{-}=A_{+}=\hat{A}^{3}=\hat{A}_{0}=\left(A^{0}-A^{3}\right) / \sqrt{2}$. Note $A^{\mu} B^{\mu}=A_{+} B_{-}+A_{-} B_{+}-A_{i} B_{i}$. Tensors are treated in a similar way and we define

$$
F_{i}=M_{i+}=\frac{1}{\sqrt{2}}\left(K_{i}-\varepsilon_{i j} J_{j}\right), \quad G_{i}=M_{i-}=\frac{l}{\sqrt{2}}\left(K_{i}+\varepsilon_{i j} J_{j}\right) .
$$

Then

$$
\begin{aligned}
& W_{i}=P_{-} \varepsilon_{i j} F_{j}-P_{+} \varepsilon_{i j} G_{j}+K_{3} \varepsilon_{i j} P_{j} \\
& W_{+}=P_{+} J_{3}+\varepsilon_{i j} P_{i} F_{j} \\
& W_{-}=-P_{-} J_{3}+\varepsilon_{i j} G_{i} P_{j} .
\end{aligned}
$$

The commutation relations of the generators are:

$$
\begin{array}{ll}
{\left[K_{3}, P_{ \pm}\right]= \pm i P_{ \pm}} & {\left[J_{3}, F_{i}\right]=i \varepsilon_{i j} F_{j}} \\
{\left[K_{3}, F_{1}\right]=i F_{i}} & {\left[J_{3}, G_{i}\right]=i \varepsilon_{i j} G_{j}} \\
{\left[K_{3}, G_{i}\right]=-i G_{i}} & \\
{\left[F_{i}, P_{-}\right]=i P_{i}} & {\left[G_{i}, P_{+}\right]=i P_{i}} \\
{\left[F_{i}, P_{j}\right]=i \delta_{i j} P_{+}} & {\left[G_{1}, P_{j}\right]=i \delta_{i j} P_{-}} \\
& {\left[F_{i}, G_{j}\right]=i \delta_{i j} K_{3}-i \varepsilon_{i j} J_{3}}
\end{array}
$$


Additionally,

$$
\begin{array}{ll}
{\left[K_{3}, W_{ \pm}\right]= \pm i W_{ \pm}} & {\left[J_{3}, W_{i}\right]=i \varepsilon_{i j} W_{j}} \\
{\left[F_{i}, W_{-}\right]=i W_{1}} & {\left[G_{i}, W_{+}\right]=i W_{i}} \\
{\left[W_{+}, W_{i}\right]=i \varepsilon_{i j}\left(P_{+} W_{j}-W_{+} P_{j}\right)} & \\
{\left[W_{-}, W_{i}\right]=i \varepsilon_{i j}\left(W_{-} P_{j}-P_{-} W_{j}\right)} & \\
\left(W_{1}, W_{2}\right]=i\left(W_{+} P_{-}-W_{-} P_{+}\right) & \\
{\left[W_{+}, W_{-}\right]=i \varepsilon_{i j} P_{i} W_{j}}
\end{array}
$$

Other commutators are zero.

The spin $\underline{s}$ and mass $\underline{M}$ defining an irreducible representation are related to the Casiair operators as follows:

$$
M^{2}=\mathrm{P}^{-2}, \quad-\mathrm{M}^{2} \mathrm{~s}(\mathrm{~s}+1)=\mathrm{W}^{-2}
$$

where $\mathrm{P}^{-2}$ and $\mathrm{W}^{-2}$ are the eigenvalues of the Casimer operators at the representation in question. It is a remarkable fact that the three quantities

$$
j_{i}=\frac{1}{\vec{M}}\left(W_{i}-\frac{W_{+}}{P_{+}} P_{i}\right), \quad j_{3}=\frac{W_{t}}{P_{+}}
$$

satisfy the commutation relations of angular momentum

$$
\left[j_{a}, j_{b}\right]=i \varepsilon_{a b c} j_{c}
$$

in the timelike case $M^{2}>0$. This form of the $j_{a}$ and their commutator follow from rest frame expressions by the Wigner boosting procedure. The details are given in Appendix A. 
After substituting for $W$ from (2.10) one can solve (2.13) to express $G_{1}, G_{2}$ and $J_{3}$ in terms of $j_{a}$.

Consequently, we obtain the universal NP form of the Poincare generators for the case $\mathrm{P}^{2}>0$,

$$
\begin{gathered}
G_{i}=\frac{1}{2}\left\{Q_{i}, P_{-}\right\}+P_{i} Q_{+}+\varepsilon_{i k}\left(M j_{k}+P_{k} j_{3}\right) / P_{+} \\
J_{3}=Q_{1} P_{2}-Q_{2} P_{1}+j_{3} .
\end{gathered}
$$

where

$$
\begin{aligned}
& Q_{1}=F_{i} / P_{+}, \quad Q_{+}=\frac{1}{2}\left\{K_{3}, 1 / P_{+}\right\} . \\
& P_{-}=\frac{P_{1} P_{i}+M^{2}}{2 P_{+}},
\end{aligned}
$$

and $\left[Q_{i}, P_{j}\right]=i s_{i j}, \quad\left[Q_{+}, P_{+}\right]=1$.

The ordering seems to be unique up to a chenge of variable, which can give the order in Ref. 26 . The ordering is post facto justified by the realization of the Poincaré group commutation relations. Natice that we may regard all the generators as given in terms of the canonical pairs $\left(Q_{+} P_{+}\right),\left(Q_{i} P_{i}\right)$, and $M$ and $j_{a}$. The latter two are in turn to be specified in terms of canonical internal coordinate pairs as will be discussed in Section IV. It is important to note that $M$ and $M^{2}$ are allowed to be operators in this formulation, contrary to the case in other formulations ${ }^{25}$ or in standard field theories. The latter are discussed in the next section. 
To understand the significance of the $j_{a}$, one should examine the Casimir cperators of the Poincaré group $P_{\mu} P^{\mu}$ and $W_{\mu} w^{\mu}$. When the former is put equal to $M^{2}$, the latter comes out $-M^{2} j_{a} j_{a}$; which shows that $j_{a} j_{a}$ is a Lorentz invariant quantity, the rest frame spin. Also $j_{3}$ is the only invariant of the sevendimensional kinematic subgroup $27\left(J_{3}, K_{3}, F_{i}, P_{i}, P_{+}\right)$known as $P_{2,2}$. 
III. Standard free field theories in null plane hamiltonian formalism

Beginning with the covariant Lagrangian for QED, others have illustrated how NP coordinates and NP $\gamma$-matrices are defined and used to completely eliminate redundant components from the Hamiltonian. 10,11 Similarly, expressions for the NP Poincaré generators without redundant components have been given for a model with interacting (psuedo-) scalar bosons and Dirac fermions. 12 We present for the first time all ten NP Poincaré generators for the vector-gluon model, which includes $Q E D$ as a special case $\left(\mathbb{m}_{\vee}=0\right)$. Only the free field expressions are given in this section; the additional interaction terms are stated in Section VI. An outline of our derivation is given in the Appendix $B$; here we have merely summarized the results.

The fermion commutation relations are

$$
\begin{aligned}
x^{-}=y^{-}: & \left\{\Psi_{\alpha}(x), \Psi_{\beta}^{+}(y)\right\}=\delta_{\alpha \beta} \delta^{3}(x-y) \quad \alpha, \beta=1,2 \\
& \left\{\Psi_{\alpha}(x), \quad \Psi_{\beta}(y)\right\}=0 \\
& \left\{\Psi_{\alpha}^{+}(x), \quad \Psi_{\beta}(y)\right\}=0
\end{aligned}
$$


where $\delta^{3}(x-y)=\delta\left(x^{+}-y^{+}\right) \delta^{2}(x-y)$, The vector field,

$$
A=\left(\begin{array}{l}
A_{1} \\
A_{2} \\
A_{3}
\end{array}\right)
$$

has the commutation relations:

$$
x^{-}=y^{-}:\left[A_{\alpha}(x), A_{\beta}(y)\right]=-\frac{i}{4} \delta_{\alpha \beta} \varepsilon\left(x^{+}-y^{+}\right) \delta^{2}(x-y), \alpha, \beta=1,2,3 .
$$

More conventional commutation relations result from the introduction of the auxiliary field $\bar{A}=\left(\bar{A}_{1}, \bar{A}_{2}, \bar{A}_{3}\right)$ defined by

$$
\bar{A}_{\alpha}(x)=i \partial_{+} A_{\alpha}(x) \text {. }
$$

Differentiation of the preceding commutator yields

$$
x^{-}=y^{-}: \quad\left[A_{\alpha}(x), A_{\beta}(y)\right]=\frac{1}{2} \delta_{\alpha \beta} \delta^{3}(x-y), \quad \alpha, \beta=1,2,3 .
$$

Taking $\mathrm{x}^{-}=0$ our results are:

$$
\begin{aligned}
& P_{i}=\int \mathrm{d}^{2} \mathrm{xdx}{ }^{+} P_{i}=\int \mathrm{d}^{2} \mathrm{xdx}{ }^{+}\left\{\Psi^{+}{ }_{i \partial_{1}} \Psi+\overline{\mathrm{A}} i \partial_{i} \mathrm{~A}\right\} \\
& P_{+}=\int d^{2} x d x^{+} P_{+}=\int d^{2} x d x^{+}\left\{\Psi^{+} i \partial_{+} \psi+\bar{A} i \partial_{+} A\right\} \\
& P_{-}=\int d^{2} x d x^{+} P_{-} \\
& =\frac{1}{4 i} \int d^{2} x d x^{+} d y^{+} \varepsilon\left(x^{+}-y^{+}\right)\left\{\Psi^{+}(x)\left(m^{2}-t^{2}\right) \Psi(y)+\bar{\Lambda}(x)\left(m_{v}^{2}-t^{2}\right) A(y)\right\}
\end{aligned}
$$




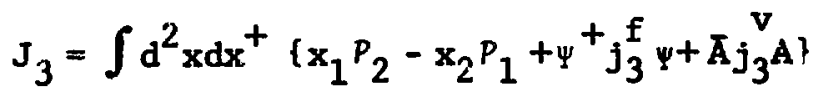

$$
\begin{aligned}
& \mathrm{K}_{3}=-\int \mathrm{d}^{2} \mathrm{xdx} \mathrm{x}^{+} \mathrm{p}_{+} \\
& F_{i}=\int d^{2} x d x^{+} x_{i} P+ \\
& G_{i}=-\int d^{2} x d x+\left\{x^{+} P_{i}-x_{i} P_{-}\right. \\
& +\frac{1}{2 i} \int \mathrm{dy}^{+} \varepsilon\left(\mathrm{x}^{+}-\mathrm{y}^{+}\right)\left[\psi^{\dagger}(x) \varepsilon_{i k}\left(j_{3}^{f_{j} \partial_{k}}+j_{k}^{f_{k}}\right) \Psi(y)\right. \\
& \left.\left.+\bar{A}(x) \varepsilon_{i k}\left(j v^{v_{i} \partial_{k}}+j_{k}^{v_{m}}\right) A(y)\right]\right\} .
\end{aligned}
$$

Here $j_{a}^{f}=\sigma_{a} / 2$ and

$$
j_{1}^{v}=\left(\begin{array}{lll}
0 & 1 & 0 \\
1 & 0 & 0 \\
0 & 0 & 0
\end{array}\right), j_{2}^{v}=\left(\begin{array}{rrr}
0 & 0 & -1 \\
0 & 0 & 0 \\
-1 & 0 & 0
\end{array}\right), j_{3}^{v}=\left(\begin{array}{rrr}
-i & 0 \\
1 & 0 & 0 \\
0 & 0 & 0
\end{array}\right)
$$

which satisfy $\left[j_{a}, j_{b}\right]=i \varepsilon_{a b c} j_{c}$. The unusual $j_{a}^{v}$ representation comes naturally from Yan's choice of fields using the "null plane gauge". ${ }^{14}$

The verification of the Poincare group commutation relations is non-trival because $A_{\alpha}\left(A_{\alpha}\right)$ does not commute with itself. Diagonalization is achieved by a Fourier transformation with . respect to $x^{+}, x^{1}, x^{2}$,

$$
\begin{aligned}
& \Psi_{\alpha}(x)=\frac{1}{(2 \pi)^{3}} \int_{-\infty}^{\infty} d^{2} p \int_{0}^{\infty} \frac{d p_{+}}{\sqrt{2 p_{+}}}\left(b_{\alpha}(p) e^{i p x}+d_{\alpha}^{\dagger}(p) e^{-i p x}\right), \\
& \psi_{\alpha}^{\dagger}(x)=\frac{1}{(2 \pi)^{3}} \int_{-\infty}^{\infty} d^{2} p \int_{0}^{\infty} \frac{d p_{+}}{\sqrt{2 p_{+}}}\left(b_{\alpha}^{\dagger}(p) e^{-i p x}+d_{\alpha}(p) e^{i p x}\right), \\
& A_{\alpha}(x)=\frac{1}{(2 \pi)^{3}} \int_{-\infty}^{\infty} d^{2} p \int_{0}^{\infty} \frac{d p_{+}}{2 p_{+}}\left(a_{\alpha}(p) e^{i p x}+a_{\alpha}^{\dagger}(p) e^{-i p x}\right),
\end{aligned}
$$


with

$$
\begin{aligned}
& \left\{b_{\alpha}(p), b_{\beta}^{\dagger}(q)\right\}=(2 \pi)^{3} 2 p_{+} \delta_{\alpha \beta} \delta^{3}(p-q) \\
& \left\{b_{\alpha}(p), b_{\beta}(q)\right\}=\left\{b_{\alpha}^{\dagger}(p), b_{\beta}^{+}(q)\right\}=0 \\
& \left\{d_{\alpha}(p), d_{\beta}^{+}(q)\right\}=(2 \pi)^{3} 2 p_{+} \delta_{\alpha \beta} \delta^{3}(p-q) \\
& \left\{d_{\alpha}(p), d_{\beta}(q)\right\}=\left\{d_{\alpha}^{+}(p), d_{\beta}^{+}(q)\right\}=0 \\
& \left\{a_{\alpha}(p), a_{\beta}^{\dagger}(q)\right\}=(2 \pi)^{3} 2 p_{+} \delta_{\alpha \beta} \delta^{3}(p-q) \\
& \left.\left\{a_{\alpha}(p), a_{\beta}(q)\right\}=a_{\alpha}^{+}(p), a_{\beta}^{\dagger}(q)\right\}=0 .
\end{aligned}
$$

Note that if $f\left(p_{+}\right)$is the $x^{+}$- Fourier transform of $f\left(x^{+}\right)$, then the transforms of

$$
\frac{i}{2} \int d y^{-} \varepsilon\left(x^{+}-y^{+}\right) f\left(y^{+}\right) \text {and }-\frac{1}{2} \int d y^{-}\left|x^{-}-y^{-}\right| f\left(y^{-}\right)
$$

are respectively $f\left(p_{+}\right) / p_{+}$and $f\left(p_{+}\right) / p_{+}{ }^{2}$. Hence, one can see by inspection that the free field generators are in the universal form of Section II. One can also observe that charge quantum numbers and other group indices may be added; they result in a mere contraction over the representation index. Additionally, there is the crucial observation that in all cases the free fields occur in a matrix contraction whose form is independent of the spin. To get different spins one adopts different representations of the $j_{a}$. The method of dynamical groups introduces harmonic oscil.lator representations of the $j_{a}$ and $\mathrm{M}^{2}$ with profound consequences. 
IV. Free field theory of trajectories

When the $j_{a}$ and $M^{2}$ have been specified in terms of a set of harmonic oscillators, we say that an internal space of hadrons has been selected. It is a Fock space spanned by oscillators $a_{n, \alpha}, a_{n, \alpha}{ }^{\dagger}$, where $\alpha$ is a charge type index and $\underline{n}$ is a generalized Poincare mode number, which has some space interpretation. The index $\underline{n}$ may be discrete, continuous, umbounded, or naturally partitioned into sets. The simplest example 25,29 contains two oscillators $a$ and $b$ with $\left[a, a^{+}\right]=1,\left[b, b^{+}\right]=1$, and

$$
\begin{gathered}
j_{+} \equiv j_{1}+i j_{2}=a b^{\dagger}, \quad j_{-} \equiv j_{1}-i j_{2}=a^{\dagger} b \\
j_{3}=\frac{1}{2}\left(a^{\dagger} a-b^{\dagger} b\right)
\end{gathered}
$$

A familiar form for: these is

$$
j_{a}=\phi^{\dagger} \frac{1}{2} \sigma_{a} \phi
$$

where

$$
\phi=\left(\begin{array}{l}
a \\
b
\end{array}\right) .
$$

The operator $M^{2}$, which must commute with $j_{a}$, is

$$
M^{2}=\frac{1}{2}\left(a^{\dagger} a+b^{\dagger} b+1\right)+m_{0}^{2} .
$$

It is true as an operator identity that

$$
J^{2} \equiv j_{a} j_{a}-\left(M^{2}-m_{o}^{2}\right)^{2}-\frac{1}{4}
$$

with

$$
j^{2}=\frac{1}{4}\left(a^{\dagger 2} a^{2}+b^{+2} b^{2}\right)+\frac{1}{2} a^{\dagger} a b^{\dagger} b+\frac{3}{4}\left(a^{\dagger} a+b{ }^{\dagger} b\right)
$$

and $m_{0}$ a constant. The rest frame space-directional significance of the oscillators is determined by their commutators with $j_{a}$. In the special model just mentioned $a$ and $b$ conotitute a two-component spinor. In general thelr comutator with $j_{a}$ may be a product of $a^{\prime} s$. Nefther $j_{a}$ nor $u^{2}$ need to be quadratic. 
In a standard field theory of spin one-half or spin-one particles the physical space contains only states $a^{\dagger}|0\rangle$ with a single oscillator acting; the representation of the Poincare group is irreducible. For example, in the gluon model of Section III terms like $\frac{1}{2} \sigma_{a}$ which act on single particle states

$$
\left(\begin{array}{l}
\psi_{1}^{+}(x) \\
\psi_{2}^{+}(x)
\end{array}\right)|0\rangle
$$

correspond to $j_{a}^{f}$, given by Eq. (4.2), which acts on single particle states

$$
\left(\Psi_{1}^{\dagger}(x) a^{+}+\Psi_{2}^{\dagger}(x) b^{+}\right)|0\rangle \text {. }
$$

The method of dynamical groups generalizes the standard theory to trajectorles by allowing any number of multiplications $\mathrm{a}^{+} \mathrm{a}^{+} \ldots \mathrm{a}^{+} \mid 0>$ in the specification of a single trajectory state. A reducible representation with all spins is thus obtained.

A second example is the well known three oscillator representation:

$$
\begin{gathered}
j_{a}=\varepsilon_{a b c} r_{b} p_{c} \\
{\left[r_{a}, p_{b}\right]=i \delta_{a b} .}
\end{gathered}
$$

A general case with $\mathbb{N}$ oscillators has been studied. 30 Here is a cubic one ${ }^{8}$ :

$$
\begin{aligned}
& j_{i}=\frac{1}{4}\left\{\left\{r_{k}, p_{k}\right\}, p_{1}\right\}-\frac{1}{4}\left\{p_{k} p_{k}-1, r_{i}\right\} \\
& j_{3}=r_{1} p_{2}-r_{2} p_{1} .
\end{aligned}
$$

The operator $\mathrm{M}^{2}$ must be rotationally invariant. (Note, with the $j_{a}$ in Eq. (4.8) the sum $r_{k} r_{k}$, for example, is not.) A function of $j_{a} j_{a}$ always works, but by no means exhausts the possibilities. 
Noteworthy is the dual model, whose cubic construction is different. There are twelve "kinds" of oscillators" for each "mode". A kind is labeled by $k(=1,2, \ldots, 12)$ and a mode by $m(x \pm 1, \pm 2, \cdots)$. Furthermore, for each pair of these there is a "transverse" label $i(=1,2)$. These oscillators

$$
\alpha_{i}^{m, k}
$$

satisfy

$$
\left[\alpha_{i}^{m, k}, \alpha_{j}^{n, \lambda}\right]=n_{\omega \delta}{ }^{m+n, o_{\delta}} \delta_{i j} \delta^{k \lambda} .
$$

Out of them we form operators

$$
\begin{aligned}
& M j_{k}=\frac{i}{2} \varepsilon_{k \ell} \sum_{m=-\infty}^{\infty} \sum_{n=-\infty}^{\infty} \sum_{k, \lambda=1}^{12} \frac{1}{n} \alpha_{\ell}^{n}, \lambda \alpha_{r}^{m, k} \alpha_{r}^{-n-m, k} \\
& \mathrm{~m} \neq 0 \quad \mathrm{n} \neq 0 \\
& m+n=0 \\
& j_{3}=-\frac{i}{\omega} \sum_{n=-\infty}^{+\infty} \sum_{k=1}^{12} \frac{1}{n} \alpha_{1}^{n, k} \alpha_{2}^{-n, k} \\
& M^{2}=-\omega+\sum_{n=1}^{\infty} \sum_{k=1}^{12} \alpha_{i}^{n, k} \alpha_{i}^{-n, k}
\end{aligned}
$$

Here $\underline{\alpha}$ is an arbitrary constant. Poincaré algebra closure is enforced through the relation

$$
\left[M j_{1}, M j_{2}\right]=i M^{2} j_{3} \text {. }
$$

The rotational property of $\alpha$ is unusual. A state is formed beginning with product type operators (omit $k$ )

$$
\alpha_{1}^{m_{1}} \alpha_{1}{ }^{m_{2}}{ }_{\alpha_{1}}^{m_{3}} \ldots \alpha_{2}{ }^{n_{1}}{ }_{\alpha_{2}}^{n_{2}}{ }_{\alpha_{2}}^{n_{3}} \ldots \text {. }
$$


A sum of such operators is required to act on $\mid 0>$ to produce a state. The relation

$$
\left[M^{2}, a^{m}\right]=\operatorname{mwa}_{j}^{m, k}
$$

shows that products of $\alpha$ with an identical superscript sum have a common mass. Only such products may, therefore, constitute the terms in a sum creating an eigenmass state. Starting, for example, with a state formed with a single product of $\alpha$ 's one may generate the partner $\left(J_{3}\right)$ states by acting with stepup operators $\mathrm{Mj}_{+} \equiv \mathrm{Mj}_{1}+i M j_{2}$ and step-down operators $M j_{-} \equiv M j_{1}-i M j_{2}$ using the relation

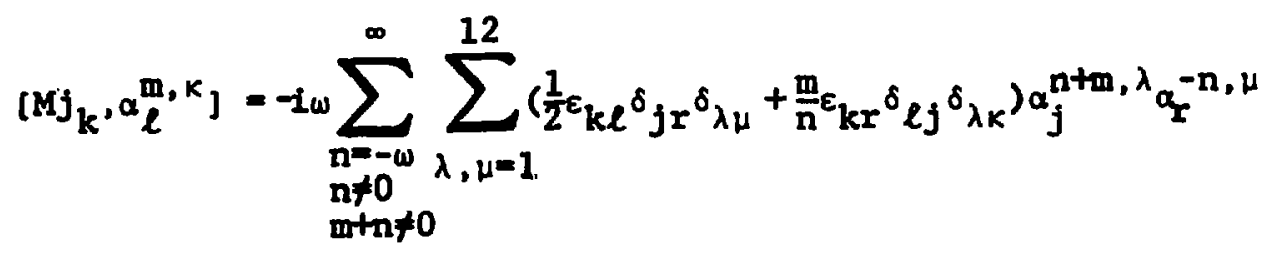

The (mass) ${ }^{2}$ of a state is the eigenvalue of $\mathrm{M}^{2}$; after finding it one may find the spin by operating with $\left(M j_{1}\right)^{2}+\left(M j_{2}\right)^{2}+M^{2} j_{3}^{2}$ to obtain $\mathrm{M}^{2} \mathrm{j}(\mathrm{j}+1)$ and thus $\mathcal{1}$ itself. We remark that, strictly speaking, the $J_{a}$ are quadratic, since $\mathrm{H}^{2}$ and $J_{3}$ are quadratic; and $\mathrm{MJ}_{1}$ are cublc in oscillators. A second remark is that the above is not exactly the dual model in twenty-81x space-time dimensions that one usually finds. Using the $\underline{k}$ variable gives a larger spectrum ${ }^{30}$, but in four dimensions, as we always require. 
Note that choosing $\mathrm{j}_{\mathrm{a}}$ and/or $\mathrm{M}^{2}$ to be of higher order in cscillator variables does not yet introduce interactions.

To match the oscillator representation there is a set of basis states with appropriate spin indices. The delta function in the commutation relations should be suitably generalized. We eschew discussion of T, C, P. ${ }^{31}$ 
v. The SU(6) $\times 0(3)$ Model.

For a more realistic choice of $\mathrm{M}^{2}$ and $\mathrm{j}_{\mathrm{a}}$ we use the quark model as a guide. The three quarks in a baryon are labeled by an index $\rho(=1,2,3)$. The $\rho^{\text {th }}$ quark is associated to a pair of oscillators $a^{p}, b^{p}$. Since there are two relative spaceposition variables for three quarks, two additional sets of three oscillators $A_{a}$ and $B_{a}(a=1,2,3)$ are required. ${ }^{33}$ These oscillators all commute with each other, and satisfy $\left[a, a^{\dagger}\right]=1$. Replace $A_{a}$ and $B_{a}$ by Hermitian combinations

$$
\begin{array}{ll}
R_{a}=\frac{1}{\sqrt{2}}\left(A_{a}+A_{a}^{\dagger}\right), & P_{a}=-\frac{i}{\sqrt{2}}\left(A_{a}-A_{a}^{+}\right) \\
r_{a}=\frac{1}{\sqrt{2}}\left(B_{a}+B_{a}^{+}\right), & P_{a}=-\frac{i}{\sqrt{2}}\left(B_{a}-B_{a}^{+}\right) .
\end{array}
$$

Then choose

$$
j_{k}=\phi^{\rho \alpha+\frac{1}{2} \sigma_{k} \phi^{\rho \alpha}}+\varepsilon_{k i j}\left(R_{i} P_{j}+r_{i} P_{j}\right)
$$

where

$$
\phi^{\rho}=\left(\begin{array}{l}
a^{\rho} \\
b^{\rho}
\end{array}\right) .
$$

A simple choice for $\mathrm{M}^{2}$ is 34

$$
M^{2}=\frac{1}{2}\left(P_{a} P_{a}+P_{a} P_{a}\right)+\frac{1}{2} \omega\left(R_{a} R_{a}+r_{a} r_{a}\right) \text {. }
$$

One opecifies that only the ground state of $a^{p}, b^{p}$ (which appear in $J_{a}$ but not in $M^{2}$ ) may be occupied. Generalizations of $M^{2}$ include spin-orbit coupling ( $\phi$ to $R$ ) and additional potentials $V\left(R_{a} R_{a}, r_{a} r_{a}\right)$; the $f_{a}$ are left unchanged. Charge type labelo may be added in the usual way to obtain a realistic hadron spectrum. Evidently the analogue of quark-anciquark mesons may 
be constructed, with any potential function of the interquark distance $\left(R_{a} R_{a}\right)$. This model now contains the spectrum but not the interactions of the usual models. It is relativistic as it stands; the correct incorporation of interactions keeping this property are yet to be found (see below).

\section{Interactions}

It has long been recognized that the introduction of an interaction changes only $P_{-}$and $G_{i}$ which acquire terms, proportional to powers of a coupling constant $\mathrm{g}$, that are cubic and higher in the fields. The free theory is recovered by putting $g=0$. Both sets of ten generators $G(g)$ and $G(0)$ must satisfy the Poincare algebra.

If we define the interaction terms $I_{-}$and $I_{1}$ by

$$
\begin{aligned}
& P_{-}(g)=P_{-}(0)+I_{-}(g) . \\
& G_{i}(g)=G_{i}(0)+I_{1}(g) .
\end{aligned}
$$

then the interaction terms for the vector-gluon model described in Appendix $B$ are

$$
\begin{aligned}
& I_{-}(g)=\int d^{2} x^{+} I_{-}(x) \\
& =\frac{8}{2} \int \mathrm{d}^{2} x d x^{+} d y^{+} \varepsilon\left(x^{+}-y^{+}\right)\left\{m_{v} \phi^{+}(x) \phi(x) A_{3}(y)-\partial_{k}\left(\phi^{+}(x) \phi(x)\right) A_{k}(y)\right. \\
& -\frac{1}{2} \phi^{ \pm}(x) A_{k}(x)\left(\partial_{k}+i \varepsilon_{k \ell} \sigma_{3}^{\partial} l^{\left.+\varepsilon_{k} \ell^{\sigma} \ell^{m}\right) \phi(y)}\right. \\
& \left.+\frac{1}{2^{\dagger}}{ }^{\dagger}(x)\left(\xi_{k}-i \varepsilon_{k \ell} \sigma_{3}{ }^{\dagger} \ell+\varepsilon_{k \ell} \ell^{m}\right) \phi(y) A_{k}(y)\right\} \\
& +\frac{g^{2}}{4 I} \int d^{2} x d x^{+} d y^{+}\left\{\varepsilon\left(x^{+}-y^{+}\right) \phi^{+}(x) A_{k}(x)\left[\delta_{k l}+1 \varepsilon_{k l} \sigma_{3}\right] A_{l}(y) \phi(y)\right. \\
& \left.-1\left|x^{+}-y^{+}\right|_{\phi}^{+}(x) \phi(x) \phi^{+}(y) \phi(y)\right\} \text {. }
\end{aligned}
$$


By means of integration by parts, the arrangement of the differentiation arrows in Eq. (6.2) is chosen so that $I_{i}$ has the particularly simple expression:

$$
I_{i}(g)=\int d^{2} x d x^{+} x_{i_{-}} I_{-}(x) \text {. }
$$

It should be stressed that these are exact expressions in $\mathrm{g}$; they just happen to be quadratic. To our knowledge only $P_{\text {_ }}(g)$ has appeared previously in the literature and then only for QED. To identify with the NP formulation of QED in Ref. 11, one puts

$$
\begin{gathered}
m_{v}=0, g=e, \quad \underline{A}=\left(A_{1}, A_{2}\right), \quad \Psi_{\text {new }}=U \Psi \\
U=\left(\begin{array}{ll}
1 & 0 \\
0 & 1
\end{array}\right)
\end{gathered}
$$

(the third component of A decouples if $m_{v}=0$. ) Also found in Ref.11 is the momentum space expression for $P_{-}, m_{v}=0$, which is discussed in terms of Feynman-like diagrams. In contradistinction to the quantities of Eq. (3.6), it is mclear how to generalize the QED expressions for $I_{-}$and $I_{i}$ to allow $\Psi$ and $A$ to have an infinite number of spin components. For example, the term $\partial_{k} A_{k}$ presumably would generalize to $\partial_{k} \Gamma^{k j m_{A}}$ where $r^{k j m}$ is some Clebsch-Gordan coefficient and the indices ( $\mathrm{jm}$ ) run over the infinite number of spin components of $A$. When $j=1, \Gamma^{k l m}=\delta^{\mathrm{km}}$. However, the value of $r^{\mathrm{kjm}}$ for other $j \neq 1$ is obscure. 
We remark that, as was also the case for $\bar{\psi} \psi \phi$ theory, the Poincaré group comnutation relations must be verified by direct tedious computation. It would be more satisfactory if one could find an operator $U$ which carries out the transformation from the set $G(0)$ to $G(g)$ :

$$
G(g)=U(g) G(0) U^{-1}(g) \text {. }
$$

Since similarity transformations preserve commutation relations, the Poincare algebra would automatically be maintained. We have tried and failed to find $U$ for QED. This is a challenging, purely mathematical problem. It is also worth-while because the solution might indicate how to construct U's for hadron theories.

An analysis of the structure of $\overline{P_{-}(g)}$ for the $\bar{\psi} \psi \phi$, gluon, and dual models shows that, in general, the interaction hamiltonian sontains a linear and a quadratic term in $g$. The linear term couples three particles at a vertex and contains either a fermion mass or the universal transverse two-vector ${ }^{35}$

$$
\vec{p}=\eta_{1} \overrightarrow{\mathrm{p}}_{2}-\eta_{2} \overrightarrow{\mathrm{p}}_{1}=\eta_{2} \overrightarrow{\mathrm{p}}_{3}-\eta_{3} \overrightarrow{\mathrm{p}}_{2}=\eta_{3} \overrightarrow{\mathrm{p}}_{1}-\eta_{1} \overrightarrow{\mathrm{p}}_{3}
$$

where for the $i-t h$ particle $p_{i}^{\mu}=\left(\frac{m_{i}^{2}+p_{i}^{2}}{2 n_{i}}, \vec{p}_{i}, n_{i}\right)$ and

$$
\overrightarrow{\mathrm{p}}_{1}+\overrightarrow{\mathrm{p}}_{2}+\overrightarrow{\mathrm{p}}_{3}=0, \quad n_{1}+n_{2}+n_{3}=0 \text {. }
$$

The coefficients are functions of $n_{1}, n_{2}, n_{3}$. Similarly, the $\mathrm{g}^{2}$ term couples four particles and depends on $\eta_{i}(i=1$ to 4$)$ with. $\sum_{i} n_{i}=0$, but it is independent of the $\vec{p}_{i}$. These terms, for the rather complex Yang-Mills Lagrangian, have been recently obtained 
by Casher. 16 Honetheless, such covariant Lagrangian theories embody no trajectory. The only existing interacting field theory with a trajectory is the dual model. Unfortunately the interaction Hamiltonian for this model has not yet been extracted, but steps have been taken in the right direction. We can at present understand completely the term linear in $\underline{e}$ and cubic in the fields by virtue of the zero slope limit concept: applied to the three-Reggeon vertex. The relevant expression $^{19,21,36}$ is

$$
\begin{aligned}
& \exp \left\{\frac{1}{2} \sum_{r, s} \sum_{n, m} N_{r s}^{n m} \vec{a}_{n}^{+r} \cdot \vec{a}_{m}^{s}\right. \\
& \left.+\sqrt{2} \vec{p} \cdot \sum_{r} \frac{1}{\alpha_{r}} \sum_{m} f_{m}\left(-\frac{\alpha_{r}+1}{\alpha_{r}}\right) a_{m}^{+r}\right\}
\end{aligned}
$$

where

$$
\mathrm{N}_{r s}^{\mathrm{nm}}=-\frac{\alpha_{1} \alpha_{2} \alpha_{3}}{\alpha_{r} \alpha_{s}} \frac{m}{n \alpha_{r}+\operatorname{mos}_{s}} f_{m}\left(-\alpha_{r+1} / \alpha_{r}\right) f_{n}\left(-\alpha_{s+1} / \alpha_{r}\right)
$$

and

$$
f_{n}(\gamma)=\frac{1}{n \gamma}(\underset{n}{n \gamma})
$$

Here $r(=1,2,3)$ labels a particle at the vertex, $\vec{a}_{n}^{r}$ is an $n$th mode oscillator, $\alpha_{r}$ is a longitudinal momentum $\left(\sum_{r=1}^{3} \alpha_{r}=0\right)$ and $\vec{p}$ is discussed above. We expand the exponential, keeping only terms containing the product of $\vec{a}_{1}{ }^{1}, \vec{a}_{1}^{2}$ and $\vec{a}_{1}^{3}$ and linear in each, thus retaining only the single excitations of the trajectory. Furthermore, we keep 
only linear terms in $\vec{p}$, noting that $f_{1}(\gamma)=1$ and find

$$
\sum_{\text {cyclic }}\left(\vec{p} \cdot \vec{a}^{1}\right)\left(\vec{a}^{2} \cdot \vec{a}^{3}\right)
$$

is the coefficient of the vertex isospin factor $\varepsilon^{\text {abc }}$ in YangMills theory. No extra factors of $n$ are present.

It remains to check the $e^{2}$ term of the dual model

Hamiltonian. It has not yet been explicitly computed, but it corresponds to the instantaneous four-string vertex ${ }^{19-20,36}$.

It should provide terms

$$
\frac{\left(n_{1}-n_{2}\right)\left(n_{3}-n_{4}\right)}{4\left(n_{1}+n_{2}\right)\left(n_{3}+n_{4}\right)} \delta_{j_{1} j_{2}} \delta_{j_{3}} j_{4}
$$

and

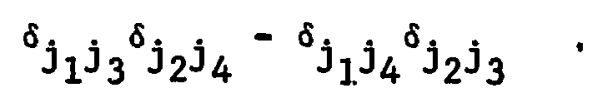

At present this has the status of a conjecture. 
VII. Conclusions

The NP Hamiltonian method has several advantages, in comparison with the infinite-component wave equation technique, which point towards its usefulness for devising an interacting trajectory theory of hadrons. The masses are all real and rising spin-mass spectra are easily accomodated, e.g., see Eq. (4.4) Also, charge ard other quantum numbers are accomodated straightforwardly. The distinction between fermions and bosons arises from the difference in commutation relations, and fermion antiparticles re indicated by an interchange of creation and annihilation operators in the momentum expansion of the field.

It is important to realize the significance of the internal oscillator coordinates in NP theories as opposed to internal (relative) Minkowski coordinates in theories of composite hadrons. The hadrons appearing in NP theories might be called "psuedocomposites" to point out the absence of a relative Minkowski coordinate together with a spin-mass multiplet structure. In both cases, the usual center-of-mass Minkowski four vector appears. Note that, in general, for a model of psuedo-composites to be relativistic, the internal oscillators must have unusual (but precise) Poincaré transformation properties.

Because no three trajectory vertex was found for the infinite-component wave equation system, no interactions of such particles are known. At the present time this is also true for the NP Hamiltonian formulation, but we have just begun to look for a vertex. If such a vertex is found it 
might lead to a NP theory of elementary psuedo-composite hadron trajectories. Perhaps it will be possible to obtain a vertex by constructing the operator $U$, of Section VI, in the form $U=\exp (i \Lambda)$ where $\Lambda$ will be cubic and quartic in the fields with coefficients which are exponential functions of the oscillators and the universal vector $\bar{p}$, as in the dual model.

We remark that if baryons are NP pseudo-composite objects, presently existing procedures for interactions and couplings, including decays and electromagnetic processes, will not be relativistic. Also, parton ideas may not be applied to them.

Acknowledgement

One of us (S.F.) thanks Dr. Tohru Eguchi for illuminating discussions and a generous interest in this work. 


\section{Appendix A}

Here we clerive, by the Wigner boosting method, the $j_{a}$ expressions used in Section II. It is well known that if $P_{\mu}\left|P^{\prime}\right\rangle=P_{\mu}^{\prime}\left|P^{\prime}\right\rangle, P_{i}|0\rangle=0$ and $P_{+}|0\rangle=2^{-\frac{1}{2}}|0\rangle$, then $\left|P^{\prime}\right\rangle$ may be expressed as

$$
\left|p^{\prime}\right\rangle=\exp \left(i p_{i}^{-} \frac{F_{i}}{p_{+}^{\prime}}\right) \exp \left(i \alpha^{\prime} k_{3}\right)|0\rangle
$$

where $e^{\alpha^{-}}=\frac{\sqrt{2} p_{+}}{M}$. Since we may diagonalize one $W_{l}$ as well as all four $P_{\mu},\left|P^{\prime}\right\rangle$ may be chosen also as an eigenstate of, say, $W_{+}$; thus we may write $\left|p^{-}, w_{+}^{-}\right\rangle$. The two-by-two representation of these boosts is familiar. Put

$$
\mathrm{J}_{\mathrm{a}}=\frac{1}{2} \sigma_{\mathrm{a}}, \quad \mathrm{k}_{\mathrm{a}}=-\frac{1}{2} \sigma_{a}
$$

and find

$$
e^{i \alpha^{\prime} K_{3}}=\left(\begin{array}{ll}
e^{\alpha^{\alpha} / 2} & 0 \\
0 & e^{-\alpha^{\prime} / 2}
\end{array}\right)=\left(\begin{array}{ll}
\sqrt{\frac{p_{+}^{\prime} \sqrt{2}}{M}} & 0 \\
0 & \sqrt{\frac{M}{p_{+} \sqrt{2}}}
\end{array}\right) \text {. }
$$

Since

$$
F_{1}=\left(\begin{array}{cc}
0 & 0 \\
\frac{-i}{\sqrt{2}} & 0
\end{array}\right), \quad F_{2}=\left(\begin{array}{cc}
0 & 0 \\
\frac{i}{\sqrt{2}} & 0
\end{array}\right)
$$

it follows that

$$
\exp \left(\frac{i}{p_{+}^{-}} p_{i}^{-} F_{i}\right)=\left(\begin{array}{ll}
1 & 0 \\
\frac{p_{1}^{i}+i p_{2}^{-}}{p_{+}^{-} \sqrt{2}} & 1
\end{array}\right)
$$


27

and that 26

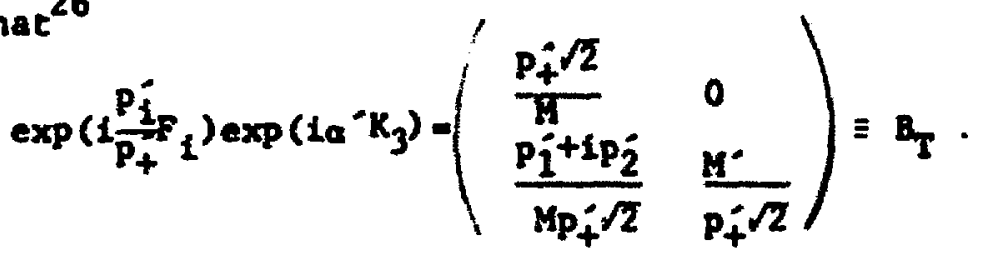

Any four vector $X_{p}$ satisfies

$$
\left[M_{\mu v}, X_{\rho}\right]=1 g_{v \rho} X_{\nu}-1 g_{\mu \rho} X_{v}
$$

and has its eigenvalues represented as a two-by two matrix

$$
x^{-}-x_{0}^{-}+x_{a}^{0} a_{a}
$$

upon which the boost acts in the form $\mathrm{B}_{\mathbf{I}} \times \mathrm{B}_{\mathbf{T}}^{+}$. The two examples at hand are $X-P$ and $X-W$. In an arbitrary frame $P$ is given by

$$
P=\left(\begin{array}{cc}
\sqrt{2 p_{+}} & p_{1}^{-i p_{2}} \\
p_{1}^{i}+i p_{2} & \sqrt{2 p_{-}}
\end{array}\right) \text {; }
$$

In the rest frame $P$ has the form

$$
\left(\begin{array}{cc}
M^{-} & 0 \\
0 & M^{-}
\end{array}\right)
$$

It is easy to see that $M^{\top} B_{T} B_{T}^{\dagger}=P$.

The equation $K_{+}=P_{+} J_{3}+\varepsilon_{1 j} P_{1} F_{j}$ shows that in this same rest frame, $w_{+}$is related to $j_{3}$ by $w_{+}^{*}=2^{-\frac{1}{4} \mathrm{H}_{3}}$ where $J_{3}|0\rangle-f_{3}|0\rangle$. Similarly $W_{i}|0\rangle=M W_{i}|0\rangle$ suggests that the set

$$
\frac{\sqrt{2}}{\sqrt{1}} w_{+} \cdot \frac{1}{w_{1}} w_{1}
$$


might satisfy the angular momentum commutation relations when they act on rest states:

$$
\begin{aligned}
& {\left[\frac{\sqrt{2} W_{+}}{M}, \frac{W_{i}}{M}\right]|0\rangle=i \varepsilon_{i j} \frac{W_{j}}{M}|0\rangle} \\
& {\left[\frac{W_{1}}{M}, \frac{W_{2}}{M}\right]|0\rangle=i \frac{W_{+} \sqrt{2}}{M}|0\rangle .}
\end{aligned}
$$

We can easily find three combinations of the Poincare generators which obey the same commutation relations when acting on boosted $\left|p^{\prime} w_{+}^{\prime}\right\rangle$ states by applying the unitary transformation

$$
U\left(P^{\circ}\right)=\exp \left(\frac{1}{P_{+}^{\prime}} P_{1} F_{1}\right) \exp \left(i \alpha^{\circ} K_{3}\right)
$$

to the $\mathrm{J}_{\mathrm{a}}$, because

$$
\begin{aligned}
\left\langle 0\left|J_{a}\right| 0\right\rangle & =\left\langle 0\left|U^{-1} \cdot U \cdot J_{a} U^{-1} U\right| 0\right\rangle \\
& =\left\langle p^{-}\left|U_{a} U^{-1}\right| p^{-}\right\rangle \\
& =\left\langle p^{-}\left|J_{a}\right| p^{-}\right\rangle
\end{aligned}
$$

Carrying out the transformation, we find Eq. (2.14). 


\section{Appendix B}

Here we sketch the derivation of Eqs, $(3,6),(6.2),(6.3)$ starting with the massive vector gluon model Lagrangian:

$$
\begin{aligned}
L= & -\frac{1}{2} B^{\mu \nu}\left(\partial_{\nu} B_{\nu}-\partial_{\nu} B_{\mu}\right)+\frac{1}{4} B^{\mu \nu} B_{\mu \nu}+\frac{1}{2} m_{v}^{2} B^{\mu} B_{\mu} \\
& +\bar{\psi}^{-}\left(\gamma^{\mu \frac{1}{2}} i{ }_{\mu}-m\right) \psi^{-}-g \bar{\psi}^{-} \gamma_{\mu} \psi^{-} B^{\mu} .
\end{aligned}
$$

Our discussion is closely related to similar work in Ref.14. The prime anticipates a particular phase transformation for $\psi$. By standard methods we construct the unsymmetrical energymomentum tensor,

$$
\hat{T}^{\mu \nu}=-\hat{g}^{\mu \nu} \nu_{L}+\hat{B}^{\lambda \mu_{\partial}} \nu_{B_{\lambda}}+\frac{i}{2} \bar{\psi}^{-} \hat{\gamma}^{\mu} \nu_{\partial}^{+} \psi_{\psi^{*}} \text {. }
$$

The meaning of the caret is given in Section II; hereafter we will drop the caret on all terms.

The field equations are

$$
\begin{aligned}
& {\left[\gamma^{\mu}\left(i \partial_{\mu}-g B_{\mu}\right)-m\right] \psi^{-}=0,} \\
& \bar{\psi}^{-}\left[\gamma^{\mu}\left(-i \partial_{\mu}^{+}-g B_{\mu}\right)-m\right]=0, \\
& B_{\mu \nu}=\partial_{\mu} B_{\nu}-\partial_{\nu} B_{\mu}, \\
& \partial_{\nu} B^{\nu \mu}+m_{v}^{2} B^{\mu}=j^{\mu},
\end{aligned}
$$

where

$$
j^{\mu}=g \bar{\psi}^{-} \gamma^{\mu} \psi^{-}
$$

In the NP there are only seven independent field components which we take to be $B_{+}, B_{+1}$, and the non-zero components of 
$\Lambda_{+} \psi^{-}$and $\bar{\psi} \Lambda_{-} \gamma^{0}=\psi^{-\dagger} \Lambda_{+}$where $\Lambda_{ \pm}$are the projection operators defined by

$$
\Lambda_{ \pm}=\Lambda^{\mp}=2^{-\frac{1}{2}} \gamma_{0} \gamma_{ \pm}=2^{-\frac{1}{2}} \gamma^{ \pm} \gamma^{0} \text {. }
$$

Thus

$$
\Lambda_{+}=\Lambda^{-}=\left(\begin{array}{llll}
1 & 0 & 0 & 0 \\
0 & 0 & 0 & 0 \\
0 & 0 & 0 & 0 \\
0 & 0 & 0 & 1
\end{array}\right), \quad \Lambda_{-}=\Lambda^{+}=\left(\begin{array}{llll}
0 & 0 & 0 & 0 \\
0 & 1 & 0 & 0 \\
0 & 0 & 1 & 0 \\
0 & 0 & 0 & 0
\end{array}\right),
$$

if

$$
\gamma^{\mu}=\left(\begin{array}{cc}
0 & -\delta_{0}^{\mu}-\delta_{a}^{\mu} \sigma^{a} \\
\delta_{0}^{\mu}+\delta_{a}^{\mu} \sigma^{a} & 0
\end{array}\right)
$$

where $\sigma^{\mathrm{a}}$ are the usual Pauli $2 \times 2$ matrices. The fleld equations split into two sets, the equations of motion of the independent field components,

$$
\begin{aligned}
& \partial_{-} B_{+}=-B_{+-}+\partial_{+} B_{-}, \\
& \partial_{-} B_{+i}=j_{i}+\partial_{+} B_{i-}+\partial_{j} B_{j i}-m_{v}^{2} B_{i} \\
& \left(i \partial_{-}-g B_{-}\right) \Lambda_{+} \psi^{-}=\gamma_{0}\left[\gamma_{k}\left(i \partial_{k}-g B_{k}\right)+m\right] \Lambda_{-} \psi^{-},
\end{aligned}
$$

and constraint equations for the redundant field components, e.g.,

$$
\left(i \partial_{+}-g B_{+}\right) \Lambda_{-} \psi^{-}=\gamma_{0}\left[\gamma_{k}\left(i \partial_{k}-g B_{k}\right)+m\right] \Lambda_{+} \psi^{\circ} \text {. }
$$

The constraint equations for the redundant vector meson components may be solved to yield 


$$
\begin{aligned}
B_{1}(x)= & \frac{1}{2} \int d^{+} E\left(x^{+}-y^{+}\right)\left[B_{+1}(y)+\partial_{1} B_{+}(y)\right] . \\
B_{+-}(x)= & \frac{1}{2} \int d^{+} E\left(x^{+}-y^{+}\right)\left[\partial_{1} B_{+1}(y)+m_{v}^{2} B_{+}(y)-j_{+}(y)\right] . \\
B_{-1}(x)= & \frac{1}{4} \int d^{+}\left|x^{+}-y^{+}\right|\left[\left(\vec{\nabla}^{2}-m_{v}^{2}\right) B_{+1}(y)-2 \partial_{1} \partial_{j} B_{+j}(y)-2 m_{v}^{2} \partial_{1} B_{+}(y)\right. \\
& \left.+\partial_{i} j_{+}(y)+\partial_{+} j_{1}(y)\right] .
\end{aligned}
$$

Here $\vec{\nabla}^{2}$ is the transverse twc dimensional Laplacian, $\varepsilon(x)=8$ ign $(x)$, and $y^{\mu}=\left(y^{+}, x^{-}, x^{L}\right)$.

The presence of the $B_{+}$term in Eq. $(B, 9)$ causes a special difficulty. It is at this point that the gauge invariance of the manifestly covariant theory is required. The usual method of dealing with the problem is to put $\mathrm{B}_{+}=0$. Another way to solve the constraint, Eq. (B.9), for $\Lambda_{-} \psi^{-}$is to introduce

$$
\psi(x)=\psi^{+}(x) e^{+g \Lambda(x)},
$$

where

$$
\Lambda(x)=\frac{1}{2} \int d y^{+} \varepsilon\left(x^{+}-y^{+}\right) B_{+}(y) .
$$

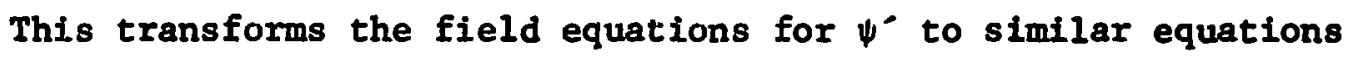
for $\psi$ in which $B_{\mu}$ is replaced by

$$
\begin{aligned}
B_{\mu}(x) & =B_{\mu}(x)-\partial_{\mu} \Lambda(x) \\
& =\frac{1}{2} \int d y^{+} \varepsilon\left(x^{+}-y^{+}\right) B_{+\mu}(y) .
\end{aligned}
$$

Note $\bar{B}_{+} \equiv 0, \quad \bar{B}_{\mu \nu}=B_{\mu \nu^{\prime}}$ and $j_{\mu}=g \bar{\psi}^{-} \gamma_{\mu} \psi^{-}=g \bar{\psi} \gamma_{\mu} \psi$.

Thus, this transformation effectively imposes the gauge condition $B_{+}=0$ in Eq. (B.9). The solution of the transformed constraint equation, for $\Lambda_{-} \psi$, is

$$
\Lambda_{-} \psi(x)=\frac{1}{2 I} \int d y^{+} \varepsilon\left(x^{+}-y^{+}\right) \gamma_{0}\left\{\gamma_{k}\left[i \partial_{k}-\bar{B}_{k}(y)\right]+m\right\} \Lambda_{+} \psi(y) .
$$


The Poincaré generators are given by Noether's theorm as

$$
\begin{aligned}
& P_{\mu}=\int d^{2} x d x x_{+\mu}^{+} \\
& M_{\mu \nu}=\int d^{2} x d x^{+}\left(x_{\mu} T_{+\nu}-x_{\nu} T_{+\mu}+B_{\sigma+} c^{\sigma \lambda} \mu \nu B_{\mu}+\frac{1}{2} v\left(\gamma_{,}^{+} \Sigma_{\mu \nu} J \psi\right)\right.
\end{aligned}
$$

where $d^{2} x=d x^{1} d x^{2}$.

$$
c^{\sigma \lambda \mu \nu}=g^{\sigma \mu_{g} \lambda \nu}-g^{\lambda \mu_{g}}{ }^{\alpha \nu} \text {. }
$$

and

$$
\Sigma^{\mu \nu}=\frac{1}{4}\left[\gamma^{\mu}, \gamma^{\nu}\right]
$$

The task now is to use the solutions of the constraint equations to eliminate all of the redundant components, $B_{1}, B_{+1}, B_{-1}$, $\Lambda_{-} \psi$, etc., and thereby obtain $P_{\mu}$ and $K_{\mu \nu}$ in a form where the integrands explicitly contain only the independent components. As an example consider

$$
\begin{aligned}
& P_{1}=\int d^{2} x d x+\left\{B_{-+} \partial_{1} B_{+}-B_{k+} \partial_{1} B_{k}+\frac{i}{2} \bar{v} \gamma_{f} \partial_{1} \psi+j_{+} \partial_{1} \Lambda\right\} \\
& =\int d^{2} x d x+\left\{\frac{1}{2} \bar{\psi}(x) \gamma_{+} y_{1} \psi+j_{+}(x) \partial_{1} \wedge(x)\right. \\
& -\frac{1}{2} \int d y^{+} \varepsilon\left(x^{+}-y^{+}\right)\left[\left(a_{k} B_{+k}(y)+m_{v}^{2} B_{+}(y)-j_{+}(y)\right) a_{1} B_{+}(x)\right. \\
& \left.\left.-B_{+k}(x) \partial_{1}\left(B_{+k}(y)+\partial_{k} B_{+}(y)\right)\right]\right\} \\
& =\int d^{2} x d x+\left(1 \sqrt{2} \psi(x) \Lambda_{+}{ }^{2}{ }_{1} \psi(x)+\frac{1}{2} \int d y^{+} \varepsilon\left(x^{+}-y^{+}\right)\left(m_{v}^{2} B_{+}(x) \partial_{1} B_{+}(y)\right.\right. \\
& \left.\left.+B_{+k}(x) \partial_{1} B_{+k}(y)\right]\right\}
\end{aligned}
$$

where surface terms coming from integration by parts have been put equal to zero.

It is convenient to define

$$
\psi=2^{\frac{k}{k}}\left(\begin{array}{l}
\psi_{1} \\
\psi_{4}
\end{array}\right), \quad \boldsymbol{\psi}^{+}=2^{\frac{1}{k}}\left(\psi_{1}^{*}, \psi_{2}^{*}\right) \text {, }
$$




$$
\begin{aligned}
& \bar{A}=\left(-1 B_{+1},-1 B_{+2},-i m_{v} B_{+}\right) \\
& A(x)=\left(\begin{array}{c}
B_{1}(x) \\
\bar{B}_{2}(x) \\
m_{v} \Lambda(x)
\end{array}\right)=\frac{1}{2} \int d y^{+} \varepsilon\left(x^{+}-y^{+}\right) A^{-T}(y) .
\end{aligned}
$$

Then $P_{i}$ takes the form given in Eq. (3.6). A more instructive example 18

$$
J_{3}=\int d^{2} x d x^{+}\left\{x_{1} T_{+2}-x_{2} T_{+1}-B_{+1} B_{2}+B_{+2} B_{1}+\frac{i}{2} \bar{\psi} \gamma_{+} \gamma_{1} \gamma_{2} \psi\right\} .
$$

By taking account of the parts integration used in the $P_{i}$ reduction and making use of the identity

$$
\bar{\psi} \gamma_{+} \gamma_{i} \gamma_{j} \psi=-\Psi^{\dagger}\left(\delta_{i j}+i \varepsilon_{i j} \sigma_{3}\right) \psi
$$

one obtains

$$
\begin{aligned}
J_{3}=\int d^{2} x d x^{+}\left\{x_{1} P_{2}-x_{2} P_{1}+\frac{1}{2} \psi^{+} \sigma_{3} \Psi\right. \\
\left.-\frac{1}{2} \int d y^{+} \varepsilon\left(x^{+}-y^{+}\right)\left[B_{+1}(x) B_{+2}(y)-B_{+2}(x) B_{+1}(y)\right]\right\} \\
=\int d^{2} x d x^{+}\left\{x_{1} P_{2}-x_{2} P_{1}+\Psi^{+} f_{3} \Psi+\bar{A}_{j}{ }_{3}^{V_{A}}\right\}
\end{aligned}
$$

where $P_{i}$ is the integrand in Eq. (3.6), $j_{i}^{f}=\frac{1}{2} \sigma_{i}$ and the $j_{i}^{v}$ are given in Section III. The other generators are obtained by similar but more lengthy calculations. 


\section{References}

1. A. O. Barut, D. Corrigan, and H. Kleinert, Phys. Rev. Lett. 20, 167 (1968).

2. Y. Hambu, Phys. Rev. 160, 1171 (1967); C. Fronsdal and L. E. Lundberg, Phys. Rev. D3, 524 (1971); A. O. Barut and w. Rasmussen, Phys. Rev. D3, 956 (1971).

3. E. Abers, I. T. Grodsky, and R. E. Norton, Phys. Rev. 159, 1222 (1967)..

4. H. Bacry and J. Nuyts, Phys. Rev. 157, 171 (1967); F. Gursey and M. Koca, Nuovo Cimento 1A, 429 (1971). .

5. S. Fubini and G. Furlan, Physics 1, 229 (1965).

6. P.A.M. Dirac, Rev. Mod. Phys. 21, 392 (1949).

7. H. Leutwyler, J. R. Klauder, and I. Streit, Nuovo Cimento 66A, $536(1970)$.

8. K. Bardakci and M. Halpern, Phys. Rev. 176, 1686 (1968).

9. R. A. Neville and J. Rohrlich, Nuovo Cimento 1A, 625 (1971).

10. J. B. Kogut and D. E. Soper, Phys. Rev. D1, 2901 (1970).

11. J. D. Bjorken, J. B. Kogut, and D. E. Soper, Phys. Rev. D3, 1382 (1971).

12. D. Flory, Phys. Rev. D1, 2795 (1970).

13. S. J. Chang and T. M. Yan, Phys. Rev. D7, 1133 (1973); S. J. Chang, R. G. Root and T. M. Yan, Phys. Rev. D7, 1147 (1973).

14. T. M. Yan, Phys. Rev. D7, 1760 and 1780 (1973).

15. E. Tomboulis, Phys. Rev. D8, 2736 (1973). 
16. A. Casher, Princeton preprint, March 1976.

17. S. J. Brodsky, R. Roskies and R. Suaya, Phys. Rev. D8, 4574 (1973).

18. The latest review is J. Scherk, Rev. Mod. Phys. 47, 123 (1975). Apologies for several hundred omissions.

19. S. Mandelstam, Nuc1. Phys. B64, 205 (1973); Phys. Rep. C13 260 (1974). .

20. M. Kaku and K. Kikkawa, Phys. Rev. D10, 1110 and 1823 (1974).

21. E. Cremmer and J. L. Gervais, Nuc1. Phys. B76, 209 (1974).

22. S. Mande1stam, Nucl. Phys. B83, 413 (1974).

23. C. Lovelace, Phys. Lett. 34B, 500 (1971).

24. P. Goddard, J. Goldstone, C. Rebbi and C. B. Thorn, Nucl. Phys. B56, 109 (1973).

25. P. A. M. Dirac, Prac. Roy. Soc. A328, 1 (1972); L. C. Biedenharn, M. Y. Han, and H. Van Dam, Phys. Rev. D8, 1735 (1973); L. P. Staunton, Phys. Rev. D8, 2446 (1973), Phys. Rev. D10, 1760 (1974).

26. H. Bacry and N. P. Chang, Ann. Phys. 47, 407 (1968).

27. F. Gursey and S. Orfanidis, Nuovo Cim. 11A, 225 (1972).

28. J. Patera, R. T. Sharp, P. Winternitz and H. Zassenhaus, J, Math. Phys. 17,977 (1976), Table VII.

29. J. Schwinger,"On Angular Momentum,"U. S. Atomic Energy Commission, N.Y.0,-3071 (1952), reprinted as"Quantum Theory of Angular Momentum" in Perspectives in Physics, edited by L. C. Biedenharn and H. Van Dam (AcademicPress, New York, 1965). 
30. V. V. Mikhailov, Teoreticheskaya i Matematichesksya Fizikn 18, 342 (1974). English translation: Theoretical and Mathematical Physics 18, 243 (1974); and previous papers.

31. P. Ramond, Nucl. Phys. B59, 412 (1973).

32. $\mathrm{E}$. P. Wigner, in Group Theoretical Concepts and Methods in Elementary Particles, edited by F. Gursey (Gordon and Breach, New York, 1963), p.37.

33. R. Horgan and R. H. Dalitz, Nuc1..Phys. B66, 1351 (1973).

34. D. Faiman and A. W. Hendry, Phys. Rev. 173, 1720 (1968); related models are in D. B. Lichtenberg, Phys. Rev. 178, 2197 (1969) and in T. Eguchi, Phys. Lett. 59B, 457 (1975).

35. See Eq. (2.30) of Ref. 20 and Eq. (3.36) of Ref. 25. 36. J. L. Torres-Hernandez, Phys. Rev. D11, 3565 (1975). 